\title{
Große Koalition - kleinkarierte und klientelorientierte Sozialpolitik?
}

\section{Ein kritischer Rückblick auf die Regierungspraxis von CDU, CSU und SPD (2005-2009)}

\section{CHRISTOPH BUTTERWEGGE}

Prof. Dr. Christoph

Butterwegge ist Hochschullehrer für Politikwissenschaft an der Universität zu Köln und Mitglied der Forschungsstelle für interkulturelle Studien (FiSt)

\author{
Das im Dezember 2013 von CDU, CSU und SPD \\ geschlossene Regierungsbündnis hat bei Teilen der \\ Bevölkerung nicht zuletzt auf sozialpolitischem \\ Gebiet große Erwartungen geweckt. Um beurteilen zu \\ können, ob diese Hoffnungen erfüllt oder enttäuscht \\ werden, ist ein Rückblick auf die von 2005 bis 2009 \\ existierende Große Koalition nützlich. Lässt man deren \\ Arbeitsmarkt-, Renten-, Familien- und Steuerpolitik \\ noch einmal Revue passieren, drängt sich der Schluss \\ auf, dass die Neuauflage der Großen Koalition auch \\ in den kommenden Jahren für die „kleinen Leute“ \\ und den Wohlfahrtsstaat wenig Gutes verheißt.
}

\section{„Nachbesserungen“ von Hartz IV: Reformmaßnahmen zur „Korrektur“ der Arbeitsmarktreform}

Weder der schwarz-rote Koalitionsvertrag noch die erste Regierungserklärung von Angela Merkel enthielten substanziell Neues zur Arbeitsmarktpolitik, die SPD und Bündnis 90/Die Grünen im Rahmen der sog. Hartz-Gesetze umfassend reformiert hatten. ${ }^{1} \mathrm{CDU}, \mathrm{CSU}$ und SPD wollten offenbar zunächst die Wirkung des rot-grünen Reformwerkes abwarten und das nach dem VW-Manager Peter Hartz benannte Gesetzespaket in Ruhe evaluieren lassen, bevor weitere Maßnahmen ergriffen werden sollten. Hans-Hermann Hartwich gelangte zu dem Ergebnis, die (nach der zwischen 1966 und 1969) zweite Große Koalition habe ,programmatisch jenseits der bisherigen grundsätzlichen Reform durch die Agenda 2010 nicht allzu viel zu bieten“ gehabt. $^{2}$
Nach dem Zweiten Gesetz zur Änderung des Zweiten Buches Sozialgesetzbuch und anderer Gesetze stieg der Regelsatz für (alleinstehende) Erwachsene in Ostdeutschland von 331 auf 345 Euro, allerdings erst zum 1. Juli 2006, was mit Softwareproblemen bei der Bundesagentur für Arbeit begründet wurde. ${ }^{3}$ Für die Sozialhilfeempfänger/innen beschloss das Kabinett am 23. August 2006 eine neue Regelsatzverordnung, mit welcher der Eckregelsatz in Ostdeutschland ab 1. Januar 2007 an die in West-

1 Vgl. hierzu und zum Folgenden ausführlicher: Christoph Butterwegge, Krise und Zukunft des Sozialstaates, 5. Aufl. Wiesbaden 2014, S. $180 \mathrm{ff}$.

2 Siehe Hans-Hermann Hartwich, „Arbeitsmarktreformen" in der Agenda der neuen Bundesregierung, in: Roland Sturm/Heinrich Pehle (Hrsg.), Wege aus der Krise? Die Agenda der zweiten Großen Koalition, Opladen/Farmington Hills 2006, S. 38

3 Vgl. hierzu und zum Folgenden: Rolf Winkel, SGB-II-Änderungsgesetz: Angleichung der Regelsätze in Ost und West - Kürzung der Leistungen für junge Erwachsene, in: Soziale Sicherheit 3/2006, S. 103 ff. 
deutschland gültige Höhe von 345 Euro angeglichen wurde. Nominal war das Arbeitslosengeld (Alg) II ursprünglich zwar höher als die Sozialhilfe, bei der allerdings erheblich mehr Sonderbedarfe über Einmalzahlungen abgedeckt wurden. Kinder unter sieben Jahren, denen bis zum 31. Dezember 2004 nur 55 Prozent des Sozialhilfe-Eckregelsatzes gewährt wurde, standen sich bei Hartz IV etwas besser, die übrigen Kinder und die Jugendlichen jedoch schlechter als früher. Dass die Große Koalition diese heimliche Kürzung nur bei den armen Schulkindern, nämlich bei den 6- bis 13-jährigen Sozialgeldbezieher(inne)n, die sie als 3. Altersgruppe neu einführte, und zwar ausgerechnet im Rahmen ihres „Konjunkturpakets II“ wieder zurücknahm, indem sie deren Transferleistung ab 1. Juli 2009 und bis zum 31. Dezember 2011 befristet von 60 auf 70 Prozent des Regelsatzes für (alleinstehende) Erwachsene auf 251 Euro anhob, zeigte zur Genüge, dass sie das Wohl der sozial Benachteiligten nicht ernsthaft im Auge hatte.

Gleichfalls mit dem Zweiten SGB-IIÄnderungsgesetz wurden Heranwachsende und junge Erwachsene zur Bedarfsgemeinschaft ihrer Eltern gerechnet und der Regelsatzbedarf für sie vom 1. April 2006 an auf 80 Prozent reduziert. Wenn diese jungen Menschen einen eigenen Hausstand gründen wollen, müssen sie nunmehr vorher die Zustimmung des kommunalen Leistungsträgers einholen. Ziehen sie ohne dessen Einwilligung bei ihren Eltern aus, erhalten sie bis zur Vollendung des 25. Lebensjahres gleichfalls nur 80 Prozent der Regelleistung. Heranwachsende und junge Erwachsene wieder in der Abhängigkeit von ihren Eltern zu belassen und ihnen per Mittelentzug die Möglichkeit der Gründung eines eigenen Hausstandes zu nehmen, ist einer wohlhabenden und hoch individualisierten Gesellschaft, die im Zeichen der Globalisierung berufliche Flexibilität und geografische Mobilität von ihren Mitgliedern verlangt, unwürdig.

Je länger das als „Hartz IV“ bekannte Vierte Gesetz für moderne Dienstleistungen am Arbeitsmarkt in Kraft war, umso deutlicher stiegen die Ausgaben für das Arbeitslosengeld II über den vom damaligen Wirtschafts- und Arbeitsminister Wolfgang Clement bewusst niedrig veranschlagten Haushaltsansatz für das Jahr 2005 hinaus - ein Trend, der sich 2006 fortsetzte und Debatten über die „Optimierung“ oder gar eine „Generalrevision“ von Hartz IV beflügelte, obwohl von einer „Kostenexplosion“ nicht die Rede sein konnte, wenn man die früher im Bereich der Sozialhilfe und des Wohngeldes anfallenden Summen mit einrechnete und berücksichtigte, dass die Ausgaben für das Arbeitslosengeld (I) durch die Verkürzung der Bezugsdauer einerseits und die Verfestigung der Langzeit- bzw. Dauerarbeitslosigkeit andererseits tendenziell sanken.

Mit dem Gesetz zur Fortentwicklung der Grundsicherung für Arbeitsuchende vom 20. Juli 2006 wurde die Beweislast bei eheähnlichen Gemeinschaften umgekehrt: Musste vorher der Leistungsträger nachweisen, dass eine solche bestand, wenn der Antragsteller und eine weitere Person länger als ein Jahr zusammenwohnten, hat dieser seit dem 1. August 2006 im Zweifelsfall den Verdacht zu widerlegen, dass es sich bei ihm und dem Mitbewohner bzw. der Mitbewohnerin um eine Bedarfsgemeinschaft handelt. Flächendeckend sollte ein Außendienst fortan prüfen, ob die Anspruchsvoraussetzungen gegeben sind, um Missbrauch vorzubeugen oder zu begegnen. Zum selben Zweck kann die Agentur für Arbeit seither Daten aus dem Kraftfahrzeugbundesamt, dem Melderegister und dem Ausländerzentralregister abrufen.

Gleichzeitig wurde die Höhe der Vermögensfreibeträge verändert: Während der Freibetrag für die Altersvorsorge von 200 auf 250 Euro pro Lebensjahr stieg, sank der Grundfreibetrag von 200 auf 150 Euro pro Lebensjahr bei entsprechender Anpassung der Höchstgrenzen, woraus Einsparungen für den Bund resultierten, weil nur geringe Sparsummen einer Zweckbindung zugunsten der Alterssicherung unterliegen. Erstantragsteller(inne)n sollte künftig sofort ein Job oder eine Fortbildung angeboten werden, um ihre Arbeitswilligkeit zu testen. Lehnt ein Antragsteller das Angebot ab oder verletzt er drei Mal seine Mitwirkungspflicht während eines Jahres, droht ihm seit dem 1. Januar 2007 ein vollständiger Leistungsentzug. Bei Jugendlichen unter 25 Jahren erstreckt sich diese Sanktion im Fall einer wiederholten Pflichtverletzung sogar auf die Kosten von Unterkunft und Heizung. Während sich dadurch die Jugendarmut noch erhöht hat, ${ }^{4}$ trug eine andere Neuregelung zur Vermehrung der materiellen
Not älterer Menschen bei: Ab 1. Januar 2007 sank der für Alg-II-Empfänger/ innen abgeführte Rentenversicherungsbeitrag von 78 auf 40 Euro pro Monat, wodurch sich die Leistungsansprüche im Alter weiter verringerten.

Die im Ersten SGB-II-Änderungsgesetz vom 22. Dezember 2005, im Zweiten SGB-II-Änderungsgesetz vom 24. März 2006 und im Hartz-IV-Fortentwicklungsgesetz vom 25. Juli 2006 enthaltenen „Korrekturen“ an der rotgrünen Arbeitsmarktreform liefen trotz einzelner Verbesserungen für die Langzeitarbeitslosen größtenteils auf eine Kürzung des Leistungsumfangs, wenn auch nicht der Regelsätze, sowie eine Ausweitung der Kontrollmaßnahmen hinaus. Damit wollte man Leistungsmissbrauch aufdecken und die Kosten für den Staat senken. Besonders problematisch war die Verschärfung der Sanktionen, zumal sich die Frage aufdrängte, ob ein vollständiger Entzug von Regelleistungen bzw. die Drohung damit nicht verfassungswidrig sind. ${ }^{5}$

Das am 1. Januar 2009 in Kraft getretene Gesetz zur Neuausrichtung der arbeitsmarktpolitischen Instrumente wickelte mit den Personal-Service-Agenturen das ursprüngliche Herzstück des Hartz'schen Reformkonzepts ab. Gleichzeitig wurden ein „Vermittlungs-“ ein „Experimentierbudget“ sowie Möglichkeiten geschaffen, Maßnahmen mit dem Ziel eines nachträglichen Erwerbs des Hauptschulabschlusses zu fördern. Indem das Gesetz bei den SGB-II-Eingliederungsleistungen den Gestaltungsspielraum für die Grundsicherungsträger vergrößerte, wurde die Rechtsposition der Fördermaßnahmen beanspruchenden Hilfebedürftigen im Zweifelsfall geschwächt.

Obwohl das Bundesverfassungsgericht die Mischverwaltung von Arbeitsagentur und kommunalen Trägern in einem am 20. Dezember 2007 verkündeten Urteil (Az.: 2 BVR 2433/04 und 2434/04) für nicht mit dem Grundgesetz vereinbar

4 Vgl. dazu: Anne Ames, Mit Druck und Strafen auf den rechten Weg bringen? - Sanktionen nach dem SGB II für Unter-25-Jährige, in: Yvonne Ploetz (Hrsg.), Jugendarmut. Beiträge zur Lage in Deutschland, Opladen/ Berlin/Toronto 2013, S. $205 \mathrm{ff}$.

5 Vgl. Annett Wunder/Alexander Diehm, SGB-II-Fortentwicklungsgesetz verschärft die Sanktionen: Sind Kürzungen des Arbeitslosengeldes II um bis zu 100 Prozent verfassungswidrig?, in: Soziale Sicherheit 6/2006, S. 195 ff. 
erklärt und eine Korrektur der Organisationsstruktur bis zum 31. Dezember 2010 verlangt hatte, konnten sich CDU, CSU und SPD bis zum Ende der Legislaturperiode nicht auf eine gemeinsame Linie einigen. Vielmehr brachte die CDU/ CSU-Bundestagsfraktion einen vom damaligen Arbeitsminister Olaf Scholz mit den beiden wichtigsten Landespolitikern Jürgen Rüttgers und Kurt Beck verabredeten und auch von Angela Merkel gebilligten Kompromiss, der eine Grundgesetzänderung zur Bestandserhaltung der Jobcenter als „Zentren für Arbeit und Grundsicherung " (ZAG) vorsah, am 17. März 2009 überraschend zu Fall. ${ }^{6}$

Um das durch die Hartz-Gesetze erleichterte Lohndumping einzudämmen, wurde der Geltungsbereich des bisher vor allem in der Bauindustrie wirksamen Arbeitnehmer-Entsendegesetzes vom 26. Februar 1996 auf die Gebäudereinigungsbranche ausgedehnt. Dagegen lehnten CDU und CSU die Einbeziehung des perspektivisch weitaus wichtigeren Leiharbeitssektors ab. Vermutlich spielte hierbei die Tatsache eine Rolle, dass der höhere Tarifabschluss des DGB und nicht der geringere des Christlichen Gewerkschaftsbundes in diesem Bereich für allgemein verbindlich erklärt worden wäre. ${ }^{7}$ Aber selbst Arbeits- und Sozialminister Franz Müntefering, der die Debatte angestoßen hatte, rang sich nicht zur Forderung nach Einführung eines allgemeinen gesetzlichen Mindestlohnes durch.

Nach zähem Ringen mit der Union, privaten Postdienstleistern wie TNT oder PIN AG und daran beteiligten Zeitungsverlegern wie der Axel Springer AG gelang es der SPD zwar im Dezember 2007, einen Mindestlohn für Briefzusteller/innen durchzusetzen. Der von dem damaligen Bundesarbeitsminister Müntefering und seinem Amtsnachfolger Scholz im zähen Ringen mit dem Koalitionspartner eingeschlagene Weg, nach entsprechenden Novellierungen des über zehn Jahre alten Arbeitnehmer-Entsendegesetzes und des sogar aus dem Jahr 1952 stammenden, früher nie angewendeten Mindestarbeitsbedingungsgesetzes für immer mehr Branchen sukzessive Lohnuntergrenzen festzulegen, war aufgrund der geringen Nachfrage auf der Arbeitgeberseite jedoch eine Sackgasse.

Bis zum Ende der von CDU, CSU und SPD vereinbarten Meldefrist (31. März 2008) hatten nur der Leih- bzw. Zeitarbeitssektor, das Wach- und Sicherheits- gewerbe sowie einige Nischenbranchen, aber nicht - wie von der SPD erhofft Schlüsselbranchen wie der Einzelhandel, die Gastronomie und die Landwirtschaft ihr Interesse an der Aufnahme ins Entsendegesetz bekundet. Die sozialdemokratische Strategie branchenspezifischer Mindestlöhne, die auf der Basis wirksamer und die Mehrheit der Beschäftigten erfassender Tarifverträge per Allgemeinverbindlichkeitserklärung durch das Bundeskabinett zustande kommen, stieß an ihre Grenze, weil sie starke Gewerkschaften dort voraussetzte, wo diese den Mindestlohn gerade aufgrund ihrer Schwäche brauchten. Das novellierte Gesetz über die Festsetzung von Mindestarbeitsbedingungen reichte nicht aus, Lohnuntergrenzen auch in Wirtschaftszweigen einzuziehen, wo (flächendeckende) Tarifverträge fehlen. Die ausgesprochen wichtige Leiharbeit wurde nicht in das Entsendegesetz aufgenommen, weil sich die Union sogar weigerte, den niedrigsten, von einer „christlichen“ (Schein-) Gewerkschaft ausgehandelten Tariflohn der Branche für allgemein verbindlich zu erklären. Stattdessen sollte eine Lohnuntergrenze für Leiharbeiter/innen im Arbeitnehmerüberlassungsgesetz vom 7. August 1972 verhindern, dass deren Löhne zu stark von den Tariflöhnen der Stammbelegschaften abweichen (sog. Equal-pay-Prinzip). Für diesen Kompromiss mit der Union verzichtete die SPD im Januar 2009 bei den Verhandlungen über das „Konjunkturpaket II“ auf eine zunächst von ihr geforderte Erhöhung der „Reichensteuer“, die eigentlich mit zu dessen Finanzierung beitragen sollte.

\section{"Generationengerechte“ Politik gegen die Rentner/innen sowie für Kinder und Jugendliche?}

Gleich zu Beginn machte die Große Koalition deutlich, dass mit Rentenerhöhungen vorläufig nicht zu rechnen sei, sondern in den nächsten Jahren weitere „Nullrunden“ anstünden. Nominale Rentenkürzungen schloss der Koalitionsvertrag zwar für die ganze Legislaturperiode aus, er sah aber zwecks Gewährleistung der Beitragssatzstabilität die Möglichkeit, „nicht realisierte Dämpfungen von Rentenanpassungen nachzuholen“, sowie die „schrittweise, langfristige Anhebung des gesetzlichen Renteneintrittsalters" vor. ${ }^{8}$ Während mit einem „Nachholfaktor“ im Rentenrecht erreicht werden sollte, dass Kürzungen, auf die zunächst verzichtet wurde, in Erhöhungsphasen letztlich doch noch weniger spektakulär - wirksam werden, wollten CDU, CSU und SPD die Lebensarbeitszeit unter Hinweis auf den demografischen Wandel verlängern und 2007 - wie im Koalitionsvertrag angekündigt - die gesetzliche Grundlage für eine 2012 beginnende und für den ersten Jahrgang bis spätestens 2035 abgeschlossene Anhebung der Regelaltersgrenze von 65 auf 67 Jahre schaffen.

Völlig unerwartet preschte der damalige Arbeits- und Sozialminister Müntefering bereits im Januar 2006 mit der Idee vor, das gesetzliche Renteneintrittsalter schneller anzuheben, als es die sog. Rürup-Kommission empfohlen und die Große Koalition vereinbart hatte: Nach dem am 1. Februar 2006 auf Drängen des Vizekanzlers vom Bundeskabinett gefassten Beschluss und seiner Übertragung in die Gesetzgebung erhöht sich das Regelrentenalter im Jahr 2012 für den Geburtsjahrgang 1947 um einen und für Folgejahrgänge jedes Jahr um einen weiteren Monat, bis der Jahrgang 1958 im Alter von 66 eine abschlagsfreie Rente ab 2024 bezieht; für die Folgejahrgänge beschleunigt sich die Anhebung der Altersgrenze um jeweils zwei Monate pro Jahr, bis der Jahrgang 1964 bereits 2031 erst mit 67 in Rente gehen kann. Nur wer mehr als 45 Jahre lang Pflichtbeiträge zur Gesetzlichen Rentenversicherung entrichtet hat, konnte seine Altersrente auch weiterhin abschlagsfrei mit 65 Jahren beziehen.

Angesichts der Tatsache, dass selbst große deutsche Unternehmen oft nur auf der Vorstandsetage noch Arbeitnehmer/ innen über 50 beschäftigen, führte das $R V$-Altersgrenzenanpassungsgesetz vom 20. April 2007 zu weiteren Rentenkürzungen, zwingt es doch mehr Beschäftigte, vor Erreichen der Regelaltersgrenze - und das heißt: mit entsprechenden

6 Vgl. Hans Nakielski, Die Neuorganisation der Grundsicherungs-Verwaltung. Bleiben Hartz-IV-Berechtigte auf der Strecke? Neues Wirrwarr von Abstimmungen und Zuständigkeiten, in: Soziale Sicherheit 5/2010, S. 168

7 Vgl. Markus Sievers, Der schwierige Kampf gegen Armutslöhne, in: Frankfurter Rundschau v. 18.8.2006

8 Siehe CDU Deutschlands/CSU Landesleitung/SPD Deutschlands (Hrsg.), Gemeinsam für Deutschland. Mit Mut und Menschlichkeit. Koalitionsvertrag von CDU, CSU und SPD, Rheinbach o.J., S. 96 
Abschlägen - in den Ruhestand zu gehen. Da half es wenig, dass Müntefering mit einer „Initiative 50plus“ zur beruflichen Wiedereingliederung älterer Langzeitarbeitsloser (Zahlung von „Kombilöhnen“ und Lohnzuschüssen an die Arbeitgeber), zur Weiterbildung älterer Arbeitnehmer/ innen (Ausgabe von Bildungsgutscheinen) sowie zur Gestaltung altersgerechter Arbeits- und Lebensbedingungen („Beschäftigungspakte“ in den Regionen) die Chancen dieser Bevölkerungsgruppe verbessern wollte.

Als die Große Koalition im April 2008 beschloss, den "Altersvorsorgefaktor“ für zwei Jahre auszusetzen und die gesetzlichen Altersrenten zum 1. Juli des Jahres um 1,1 Prozent statt nur um 0,46 Prozent anzuheben, erhob sich im deutschen Blätterwald ein Sturm der Entrüstung, obwohl die beiden Stufen der sog. Riester-Treppe 2012/13 nachgeholt werden sollten. Eine ähnliche mediale Überreaktion gab es, als CDU, CSU und SPD auf Initiative des damaligen Arbeitsund Sozialministers Scholz im Vorfeld des Bundestagswahlkampfes 2009 eine Schutzklausel beschlossen, um angesichts erstmals nach dem Zweiten Weltkrieg sinkender Bruttolöhne zumindest eine nominale Rentenkürzung auszuschließen und den Ruheständler(inne)n die Angst davor zu nehmen. In den Folgejahren wurden an sich fällige Rentenerhöhungen allerdings mit den nicht ausgefallenen, sondern nur aufgeschobenen Kürzungen verrechnet.

CDU, CSU und SPD hielten unbeirrt an der Generallinie ihrer rot-grünen Vorgängerregierung fest, die Finanzierungsgrundlagen des Sozialversicherungssystems durch materielle Zugeständnisse gegenüber den Arbeitgebern systematisch zu untergraben, worunter seine Akzeptanz bei den Arbeitnehmer(inne)n leidet, was es wiederum „anfälliger“ für Reformmaßnahmen macht. Winfried Schmähl, der fürchtet, dass die Altersrenten in ihrem Realwert wie in Relation zum allgemeinen Einkommensniveau weiter zurückbleiben, nennt in diesem Zusammenhang den Beschluss der Großen Koalition, die als befristete Anschubfinanzierung für die private Altersvorsorge gedachte abgabenfreie Entgeltumwandlung als Dauerregelung beizubehalten. SPD und Bündnis 90/ Die Grünen hatten den Versicherten mit der Riester-Reform bis zum 31. Dezember 2008 befristet das Recht eingeräumt, Teile ihres Lohns in - ausschließlich von den Beschäftigten finanzierte-Ansprüche auf betriebliche Altersrenten umzuwandeln, ohne dass Steuern und Sozialabgaben für diese Lohnanteile anfielen. Davon profitieren die Arbeitgeber, während die Einnahmenbasis der Rentenversicherungsträger unterminiert und der Leistungsanspruch aller Versicherten reduziert wird. ${ }^{9}$

Die am 1. September 2006 in Kraft getretene Föderalismusreform war der schwerwiegendste Eingriff in das Grundgesetz seit den Notstandsgesetzen. Durch die größte Verfassungsänderung seit 1949 wurden die sozialpolitischen Kompetenzen des Zentralstaates ebenso beeinträchtigt wie die Einflussmöglichkeiten der ärmeren Bundesländer. Kaum war das Grundgesetz geändert, schon standen in einigen Ländern soziale Mindeststandards zur Disposition: Nordrhein-Westfalen, seinerzeit von CDU und FDP regiert, sowie Berlin, damals von SPD und Linkspartei.PDS regiert, liberalisierten als erste Bundesländer den Ladenschluss und ermöglichten damit (großen) Geschäften nunmehr Öffnungszeiten rund um die Uhr; bloß der Sonntag und gesetzliche Feiertage blieben - wohl aus Gründen christlicher Pietät, nicht des Arbeitsschutzes und der nötigen Erholungspausen für die Verkäufer/innen wegen - von dieser Freigabe verschont. Das jahrzehntelang CDU-dominierte Baden-Württemberg wiederum preschte bei den Pensionsregelungen für seine Beamt(inn)en vor; die Verlängerung von deren Lebensarbeitszeit avancierte praktisch über Nacht zu einem „Standortvorteil“ für ihren Dienstherrn. Umgekehrt verhielt es sich beim längst überfälligen Nichtraucherschutz in Kneipen, Restaurants und Diskotheken: Mit der Begründung, dass nur die einzelnen Länder entsprechende Rauchverbotsbestimmungen für diesen Bereich erlassen könnten, setzten die Tabak- und die Gaststättenlobby im Dezember 2006 gemeinsam durch, dass kein Bundesgesetz zustande kam, obwohl sich die Große Koalition darauf in einer gemeinsamen Arbeitsgruppe bereits geeinigt hatte und der immer noch auf zentraler Ebene zu regelnde Arbeitsschutz eine Handhabe dafür bot.

Im Rahmen der Föderalismusreform II sorgten CDU, CSU und SPD mittels Einfügung der „Schuldenbremse“ ins Grundgesetz dafür, dass die jährliche Nettokreditaufnahme des Bundes ab 2016 auf 0,35 Prozent des Bruttoinlandsprodukts begrenzt wurde, während eine
Neuverschuldung der Bundesländer ab 2020 bloß noch im Falle außergewöhnlicher Umstände (Naturkatastrophen, Finanzmarktkrisen usw.) erlaubt ist. Berücksichtigt man, dass die Sozialausgaben wegen der zunehmenden Probleme auf diesem Gebiet in den Haushalten von Bund und Ländern perspektivisch einen wachsenden Anteil beanspruchen, dürfte diese Form einer Kreditsperre der öffentlichen Hand für die Sozialpolitik im föderalen Staatswesen katastrophale Folgen haben.

\section{Familien- als Bevölkerungspolitik: Kampf der Armut an statt der Armut von Kindern}

In der Familienpolitik bewegten sich CDU/CSU und SPD seit geraumer Zeit aufeinander zu, was es Ursula von der Leyen, die ein hohes Maß an Kontinuität gegenüber ihrer sozialdemokratischen Amtsvorgängerin Renate Schmidt verkörperte, in dieser Funktion ermöglichte, eine Vielzahl öffentlichkeitswirksamer Initiativen zu entfalten. So konnte die Familienministerin schnell mit Erfolgen aufwarten, ohne erst im Rahmen einer inhaltlichen Neuausrichtung ihres Hauses nach tragfähigen Kompromissen suchen zu müssen. ${ }^{10}$ Annette Henninger und Angelika von Wahl machen dafür neben Ursula von der Leyens „Führungsstärke“ ihre Fähigkeit verantwortlich, die Arbeitgeber als Bündnispartner zu gewinnen, indem sie angestrebte Reformmaßnahmen ökonomisch und demografisch begründete, also auf das brachliegende Arbeitskräftepotenzial hoch qualifizierter Mütter hinwies und Kinder als wertvolles „Humankapital“ in den Blick nahm. ${ }^{11}$

9 Vgl. Winfried Schmähl, Vergangenheit, Gegenwart und Zukunft der Gesetzlichen Rentenversicherung: Verhinderung von Armut im Alter?, in: Christoph Butterwegge/Gerd Bosbach/Matthias W. Birkwald (Hrsg.), Armut im Alter. Probleme und Perspektiven der sozialen Sicherung, Frankfurt am Main/ New York 2012, S. 52 f.

10 Vgl. Nancy Ehlert, Die Familienpolitik der Großen Koalition, in: Sebastian Bukow/ Wenke Seemann (Hrsg.), Die Große Koalition. Regierung - Politik - Parteien 2005-2009, Wiesbaden 2010, S. 157

$11 \mathrm{Vgl}$. Annette Henninger/Angelika von der Wahl, Das Umspielen von Veto-Spielern. Wie eine konservative Familienministerin den Familialismus des deutschen Wohlfahrtsstaates unterminiert, in: Christoph Egle/Reimut Zohlnhöfer (Hrsg.), Die zweite Große Koalition. Eine Bilanz der Regierung Merkel 20052009, Wiesbaden 2010, S. 362 und 368 f. 
Auf ihrer Klausurtagung im Schloss Genshagen konkretisierte die Bundesregierung zu Beginn des Jahres 2006 ihre Ankündigung, die steuerliche Absetzbarkeit von Kinderbetreuungskosten zu verbessern. Bereits eine gute Woche später stellte die SPD den Konsens jedoch wieder in Frage, weil sie unter Hinweis auf die Situation von Alleinerziehenden und Geringverdiener(inne)n die Anerkennung der Betreuungskosten vom ersten Euro an durchsetzen wollte. Der am 31. Januar 2006 zwischen den Spitzen der Fraktionen von Union und SPD gefundene Kompromiss war noch nicht das letzte Wort, der Streit um die genaue Ausgestaltung der Reform ging vielmehr weiter. Erst nach zähen Verhandlungen zwischen dem sozialdemokratischen Finanzminister und der Familienministerin sowie führenden Repräsentant(inn)en von CDU, CSU und SPD einigte man sich: Alleinerziehende und zusammenlebende Paare, die beide berufstätig sind und sich als Doppelverdiener/innen eine Tagesmutter oder Kinderfrau für ein im Haushalt lebendes Kind leisten können, das jünger als 14 Jahre ist, dürfen die Aufwendungen dafür zu zwei Dritteln bis zum Höchstbetrag von 4.000 Euro als Werbungskosten bzw. Betriebsausgaben absetzen. Außerdem können Alleinverdienerpaare mit Kindern, die zwar das 3., aber noch nicht das 6 . Lebensjahr vollendet haben, nunmehr zwei Drittel der Kinderbetreuungskosten bis zum selben Höchstbetrag als Sonderausgaben vom Gesamtbetrag ihrer Einkünfte abziehen.

Während sozial benachteiligte Familien, die aufgrund eines fehlenden oder zu geringen Einkommens keine Steuern zahlen, überhaupt nicht in den Genuss dieser Regelungen kommen, die bezeichnenderweise Teil des Gesetzes zur steuerlichen Förderung von Wachstum und Beschäftigung vom 26. April 2006 waren, profitieren Besserverdienende überdurchschnittlich davon. Ähnliches gilt für das Elterngeld, welches am 1. Januar 2007 das Erziehungsgeld ablöste: Obwohl der einkommensabhängige und erst bei 1.800 Euro pro Monat gedeckelte Geldbetrag in Höhe von 67 Prozent des Nettoerwerbseinkommens jenes Elternteils, der das Kind unter vorübergehender Aufgabe oder Reduktion seiner Berufstätigkeit auf höchstens 30 Wochenstunden betreute, zunächst nicht - wie im Koalitionsvertrag angekündigt und von der CSU verlangt - auf die Sozialhilfe bzw. das
Arbeitslosengeld II angerechnet wurde, hatten deren Bezieher/innen, Studierende und Geringverdiener/innen (darunter überdurchschnittlich viele Frauen), die Kinder bekamen, hiervon ausschließlich Nachteile. Denn bisher war ihnen das Erziehungsgeld in Höhe von 300 Euro pro Monat zwei Jahre lang oder als sog. Budget in Höhe von 450 Euro ein Jahr lang gezahlt worden. Elterngeld gab es dagegen nur für ein Jahr, sieht man davon ab, dass Erwerbstätigen unter bestimmten Voraussetzungen zwei (Partner-)Monate zusätzlich gewährt werden; sein Sockelbetrag, mit dem Studierende, Sozialhilfebezieher/innen, Arbeitslose und Geringverdiener/innen auskommen mussten, lag überdies bloß bei 300 Euro.

Mithin erhalten Gutbetuchte auf Kosten der sozial Benachteiligten mehr (Eltern-)Geld, das vor allem hoch qualifizierte, gut verdienende Frauen motivieren soll, Kinder zu bekommen und anschließend schnell wieder in den Beruf zurückzukehren. Das Elterngeld ist ein sozialpolitisches Paradox, weil der Staat damit - wie im Falle steuerlicher Kinderfreibeträge - ausgerechnet jene Anspruchsberechtigten am meisten subventioniert, die es am wenigsten nötig haben. Dass mit dem Elterngeld eine qualitative Bevölkerungspolitik gemacht werden sollte, bestätigte die Begründung der Leistung im gemeinsamen Entwurfstext der Koalitionsfraktionen: „Deutschland hat mit 1,36 Kindern pro Frau (...) eine der niedrigsten Geburtenraten der Welt. Mehrkindfamilien werden seltener, und die Kinderlosigkeit in Deutschland ist hoch. Besonders auffällig ist, dass in Deutschland insgesamt 39 Prozent der 35- bis 39-jährigen Akademikerinnen ohne Kinder im Haushalt leben. "12

Ursula von der Leyen vertrat die Auffassung, dass sie als Ministerin eine nachhaltige, „bevölkerungsbewusste“ Familienpolitik machen und die Sozialpolitik im Sinne einer Arbeitsteilung zwischen beiden Ressorts für die Bedürftigen sorgen müsse. Dabei ist eine Familienpolitik, die man als unsozial bezeichnen kann, gar keine Familienpolitik, weil sie es einem Großteil junger Menschen gerade nicht ermöglicht, ihren Kinderwunsch zu verwirklichen. Indem die Honorierung von Betreuungs- und Erziehungsleistungen der Eltern stärker als bisher akzentuiert wird, avanciert die Familienpolitik zum Hauptexerzierfeld für Leistungsgerechtigkeit als Ersatz für die immer weniger gewährleis- tete, aber für den Sozialstaat konstitutive Bedarfsgerechtigkeit. Anuscheh Farahat, Stefanie Janczyk, Annett Mängel und Barbara Schönig sprachen mit Blick auf die Frauen- und Familienpolitik der Großen Koalition von „exklusiver Emanzipation“, was sie wie folgt begründeten: „In Familien mit niedrigem Haushaltseinkommen, für Arbeitslose, Studierende und Alleinerziehende wird das Elterngeld die bestehenden geschlechtsspezifischen Rollen und die soziale Lage keineswegs verbessern, sondern verschlechtern.“"13 Offenbar wollte man damit weniger die Armut von Kindern als die Armut an Kindern bekämpfen.

Gegenüber den Armen zeigten sich CDU, CSU und SPD nicht eben großzügig: Als die Koalition rechtzeitig vor dem Jahresende 2008 beschloss, vom Beginn des Folgejahres an das Kindergeld für das erste und zweite Kind um 10 Euro und ab dem dritten Kind um 16 Euro pro Monat zu erhöhen, einigte man sich auf Initiative der SPD gleichzeitig darauf, für die Kinder von Hartz-IV-Bezieher(inne)n, die nicht in den Genuss des höheren Kindergeldes kommen, weil es voll auf ihre Transferleistung angerechnet wird, ein „Schulbedarfspaket“ in Höhe von 100 Euro pro Schuljahr zu schnüren. Es sollte nach dem am 1. Januar 2009 in Kraft getretenen Familienleistungsgesetz allerdings nur bis zur 10. Klasse gewährt werden. CDU und CSU hatten auf dieser Begrenzung bestanden, weil die SPD ihrem Wunsch nach Steuerprivilegien für Eltern, deren Kinder auf Privatschulen gehen, nicht entsprach. Die öffentliche Kritik an der beschlossenen Regelung blieb nicht aus, schien es doch geradezu so, als wollte die Große Koalition damit unterstreichen, dass die Kinder aus sozial benachteiligten Familien kein Abitur machen sollen, oder dokumentieren, dass Gymnasiasten der höheren Klassenstufen ohnehin aus Elternhäusern kommen, die keiner staatlichen Zuwendung bedürfen. Auf einer Sitzung des Koalitionsausschusses am 4./5. März 2009 verständigten sich CDU, CSU und SPD schließlich darauf, den Gesetzestext an diesem Punkt nachzubessern und auch Oberstufenschüler/innen und

12 Gesetzentwurf der Fraktionen der CDU/ CSU und SPD: Entwurf eines Gesetzes zur Einführung des Elterngeldes, BT-Drs. $16 / 1889$ v. 20.6 .2006 , S. 33

13 Anuscheh Farahat u.a., Exklusive Emanzipation. Zur Frauen- und Familienpolitik der großen Koalition, in: Blätter für deutsche und internationale Politik 8/2006, S. 985 
Vollzeit-Berufsschüler/innen sowie die Kinder von Geringverdiener(inne)n in den Genuss des „Schulbedarfspaketes“ kommen zu lassen, das jedoch den realen Bedarf nur unzureichend deckte.

Im Kinderförderungsgesetz vom 10. Dezember 2008 wurde festgelegt, dass Kinder ab 1. August 2013 nach Vollendung des ersten Lebensjahres einen Rechtsanspruch auf den Besuch einer Tageseinrichtung oder Förderung in Kindertagespflege haben, die stark ausgebaut werden und 30 Prozent der neuen Betreuungsplätze stellen sollte. Vom selben Zeitpunkt an sollten Eltern, die

\section{Die SPD unternahm im Bundestagswahlkampf eine Kehrtwende, die man als steuerpolitische Springprozession bezeichnen kann.}

ihre ein- bis dreijährigen Kinder nicht in Einrichtungen betreuen lassen wollen oder können, eine monatliche Zahlung erhalten. Vor allem die CSU forderte die Einführung eines Bundesbetreuungsgeldes in Höhe von 150 Euro pro Monat, während SPD-Politiker/innen von einer „Herdprämie“ sprachen, weil es viele Mütter veranlassen könnte, nicht (mehr) berufstätig zu sein, wodurch das traditionelle „Alleinernährer“-Familienmodell zementiert würde. Daran änderte auch der diskriminierend wirkende Plan nichts, Transferleistungsempfänger(inne)n kein Bargeld auszuzahlen, sondern ihnen im Unterschied zu den übrigen Eltern, die lieber zu Hause bleiben, als erwerbstätig zu sein, (Bildungs-)Gutscheine für ihr Kind zu geben. ${ }^{14}$

\section{Steuerpolitik als Echternacher Springprozession}

Ein zentraler Streitpunkt des an kontroversen Themen armen Bundestagswahlkampfes 2005 war die künftige Höhe der Mehrwertsteuer. CDU und CSU vertraten die Auffassung, der gültige Regelsatz von 16 Prozent müsse um zwei Punkte erhöht werden, um mit den höheren Staatseinnahmen die „gesetzlichen Lohnnebenkosten" senken und den „Faktor Arbeit“ entlasten zu können. Während sich die christdemokratische Kanzlerkandidatin mit der Begründung einer größeren Ehrlichkeit und Seriosität rühmte, sie kündige eine solche Steuererhöhung bereits vor der Wahl an, machten SPD-Politiker mit dem Schimpfwort „Merkel-Steuer“, die sie auf Plakaten und in Reden zu stoppen versprachen, massiv Stimmung dagegen. Umso erstaunter war man, als die Vertreter/innen von CDU, CSU und SPD während ihrer Koalitionsverhandlungen nicht etwa eine moderate Erhöhung der Mehrwertsteuer um einen Prozentpunkt, sondern zum 1. Januar 2007 eine Steigerung von 16 auf 19 Prozent ankündigten. Das war für große Familien, besonders jene von Geringverdiener(inne)n, die einen Löwenanteil ihres Einkommens in den Konsum stecken (müssen), eine spürbare finanzielle Mehrbelastung. Denn sie trifft die Mehrwertsteuer naturgemäß stärker als Besserverdienende (ohne Kinder), wiewohl indirekte bzw. Verbrauchssteuern generell unsozial erscheinen, weil sie die materielle Lage und die finanzielle Leistungsfähigkeit der Steuerpflichtigen nicht berücksichtigen.

Nachdem die rot-grüne Koalition den Spitzensteuersatz während ihrer siebenjährigen Regierungszeit um elf Punkte auf 42 Prozent gesenkt hatte, unternahm die SPD im Bundestagswahlkampf 2005 eine moderate Kehrtwende, die man auch als steuerpolitische Springprozession bezeichnen kann. Sie forderte nämlich einen werbewirksam als „Reichensteuer“ titulierten Aufschlag in Höhe von drei Prozentpunkten auf die Einkommensteuer für Alleinstehende ab einem Jahreseinkommen von 250.000 Euro bzw. 500.000 Euro für gemeinsam veranlagte Ehepaare. CDU und CSU setzten im Koalitionsvertrag durch, dass die gewerblichen Einkünfte ihrer wohlhabendsten Klientel bei der Erhebung dieser neuen Steuer unberücksichtigt blieben. Aufgrund verfassungsrechtlicher Bedenken gegenüber der Ungleichbehandlung von Einkunftsarten wurden schließlich alle Gewinneinkünfte (aus Landwirtschaft, Gewerbebetrieb oder selbstständiger Arbeit) ausgenommen.

Mit dem Steueränderungsgesetz 2007 vom 19. Juli 2006 wurden die Zuweisungen des Bundes an die Gesetzliche Krankenversicherung mit der Perspektive abgesenkt, sie im Jahr 2008 auslaufen zu lassen. Während der Bundeszuschuss an die Agentur für Arbeit ganz wegfiel, sollte die Dynamik der Zuschüsse an die Gesetzliche Rentenversicherung gestoppt werden. Außerdem wurde die Entfernungspauschale für Fahrten von der Wohnung zum Arbeitsplatz bis 20 $\mathrm{km}$ Entfernung gestrichen, der Sparerfreibetrag von 1.370 auf 750 Euro für Ledige und von 2.740 auf 1.500 Euro für Ehepaare fast halbiert und die Zahlung von Kindergeld für noch in der Ausbildung befindlichen Nachwuchs auf die Zeit bis zum vollendeten 25. (bislang: 27.) Lebensjahr begrenzt. Kosten für ein häusliches Arbeitszimmer konnten steuerlich nur noch geltend gemacht werden, wenn es den Mittelpunkt der beruflichen Tätigkeit bildet. Gerade die leistungsbereiten Mittelschichten, deren Interessen zu vertreten Kurt Beck als damaliger SPD-Vorsitzender seiner Partei riet, wurden durch die schwarz-rote Regierungspolitik einmal mehr getroffen, nämlich geografisch mobile Berufspendler/innen, Eigenheimbesitzer und Lehrer/ innen. Teilweise mussten die Regelungen der Großen Koalition auf den genannten Feldern später wieder modifiziert oder sogar zurückgenommen werden: Sowohl die bei der Kilometerpauschale getroffene Regelung, sie erst von einer bestimmten Distanz zwischen Wohnung und Arbeitsplatz an zu zahlen, wie auch die Streichung der Möglichkeit für Lehrer/innen, ein Arbeitszimmer steuerlich abzusetzen, obwohl ihnen an der Schule kein Dienstraum zur Verfügung stand, erklärte das Bundesverfassungsgericht für mit dem Grundgesetz nicht vereinbar.

Durch die Senkung des Körperschaftsteuersatzes auf 15 Prozent entließen CDU, CSU und SPD das Großkapital weitgehend aus seiner Verantwortung für die Finanzierung des Gemeinwesens. Dass dieser Satz zu Beginn der „Ära Kohl“ 1982 noch 56 Prozent und zu Beginn der Amtszeit von Bundeskanzler Gerhard Schröder 1998 noch 53 Prozent betragen hatte, zeigt nur zu deutlich, wie sehr die Kapitalgesellschaften in der Folgezeit entlastet wurden. Einkommen aus Vermögen und Kapitalerträge (Zinsen, Dividenden und Veräußerungsgewinne, die früher nach der zwölfmonatigen Spe-

14 Vgl. ergänzend hierzu: Christoph Butterwegge, Vorwärts in die Vergangenheit? Refamiliarisierung und Reprivatisierung der Kinderbetreuung, in: Klaus Hurrelmann/ Tanjev Schultz (Hrsg.), Staatshilfe für Eltern. Brauchen wir das Betreuungsgeld? Weinheim/Basel 2013, S. 158 ff. 
kulationsfrist steuerfrei blieben) wurden gegenüber anderen Einkunftsarten privilegiert, d.h. Rentiers niedriger als Arbeitnehmer/innen besteuert. An die Stelle der Zinsabschlag- trat eine pauschale Abgeltungssteuer auf Kapitalerträge in Höhe von 25 Prozent, die vornehmlich den Spitzensteuersatz bei der Einkommensteuer zahlenden Personen zugute kommt, während Kleinanleger/innen dadurch (zunächst) sogar stärker belastet werden können, und trotz Verbreiterung der Bemessungsgrundlage zu Steuerausfällen geführt hat.

Vergleichbares vollzogen CDU, CSU und SPD im Erbschaftsteuerrecht. Während die Armut allmählich bis zur Mitte der Gesellschaft vorstieß, ${ }^{15}$ machte die Große Koalition dem Nachwuchs von Millionären, Multimillionären und Milliardären weitere Steuergeschenke. Wer als Witwe oder Waise einen Familienkonzern à la Burda, Oetker oder Springer erbte, ihn zehn Jahre lang nicht veräußerte und die Bruttolohnsumme konstant hielt, wurde von der betrieblichen Erbschaftsteuer befreit.

\section{Fazit und Schlussfolgerungen}

Da die Zweidrittelmehrheit von CDU/ CSU und SPD kaum Raum für Erfolg versprechende Initiativen der Opposition ließ und sich im Parlament nicht mehr zwei fast gleich starke Machtblöcke gegenüberstanden, erlahmte die öffentliche Debatte über die Zukunft des Wohlfahrtsstaates mit Bildung der zweiten Großen Koalition. Auch die journalistischen Meinungsführer verkeilten sich unabhängig von ihrer „Lagerzugehörigkeit" nicht mehr so wie früher ineinander, was auf den Parteienstreit mäßigend wirkte, die sozialpolitischen Kontroversen aber weniger spannend machte. Außerparlamentarisch gab es eher weniger Massenbewegungen und seltener Aktionen wie die sich allmählich verlaufenden Montagsdemonstrationen gegen Hartz IV bzw. „Sozialraub“ und „Rentenklau“, denn die an der Regierung beteiligten Volksparteien banden einen Großteil des Protestpotenzials an sich oder neutralisierten es zumindest weitgehend.

Die schwarz-roten Reformen waren von zahlreichen Brüchen und Widersprüchen gekennzeichnet. Weder ließ sich in der Sozialpolitik eine klare Linie erkennen, noch wurde sie konsequent durch- gehalten. Da suchte man beispielsweise mit den Sozialversicherungsbeiträgen die „Lohnnebenkosten“ zu senken, kompensierte aber die Senkung der Beiträge zur Arbeitslosenversicherung zum 1. Januar 2007 durch eine nur unwesentlich geringere Erhöhung der Rentenversicherungs- und Krankenkassenbeiträge. Man wollte „versicherungsfremde Leistungen“ wie die beitragsfreie Mitversicherung der Kinder stärker über Steuern finan-

\section{$C D U, C S U$ und SPD vergrößerten die soziale Schlagseite der Regierungspolitik: Statt Armut zu bekämpfen, bekämpften sie die Armen.}

zieren, strich den Krankenkassen aber gleichzeitig bisher gewährte Zuschüsse aus der Tabaksteuer und erhöhte ihre laufenden Kosten durch die Anhebung der Mehrwertsteuer. Der damalige Vizekanzler Müntefering, im politischen Bermudadreieck zwischen Mindestlöhnen, sog. Kombilöhnen und Zuverdienstgrenzen beim Alg II steckend, dachte laut über deren Senkung nach, um die große Zahl der „aufstockenden“ Geringverdiener/innen einzudämmen, obwohl die seinerzeit noch von ihm selbst geführte Mehrheitsfraktion des Bundestages die entsprechenden Freibeträge erst zum 1. Oktober 2005 angehoben hatte, um Langzeitarbeitslosen größere finanzielle Anreize zur Arbeitsaufnahme zu geben.

Gegner einer fortschrittlichen und finanziell aufwendigen Sozialpolitik warfen der zweiten Großen Koalition fehlenden Mut, Reformunfähigkeit und politischen Immobilismus vor. Manfred G. Schmidt kritisiert, „dass sie ihren potentiell großen politischen Spielraum und die Möglichkeit, den politischen Protest, den unpopuläre Reformen hervorbringen würden, gemeinsam zu schultern, nicht zu beherzteren Sanierungsreformen am deutschen Wohlfahrtsstaat nutzte."16 Zwar erschien die schwarz-rote Reformpolitik weniger spektakulär als die von Peter Hartz inspirierte Arbeitsmarktreform, wirkte subtiler als diese und hatte manchmal auch stärker Stückwerkcharakter, war aber genauso unpopulär und keineswegs sozial gerechter. Denn die CDU/CSU/SPD-Regierung verteilte großzügige Steuergeschenke an die „obe- ren Zehntausend“, während sie vor allem gegenüber der Unterschicht zugeknöpft und kleinkariert agierte.

Obwohl eine Koalition der großen „Volksparteien“ ihrer ganzen Konstruktion wie der unterschiedlichen programmatischen Tradition aller Beteiligten nach den Eindruck vermittelt, sämtliche Bevölkerungsschichten seien mit ihren spezifischen Interessen angemessen repräsentiert, vergrößerten CDU, CSU und SPD die soziale Schlagseite der Regierungspolitik. Statt die Armut zu bekämpfen, bekämpften sie die Armen. Die „kleinen Leute“ gehörten zu den Verlierer(inne)n der Großen Koalition: Hauptleidtragende ihrer Politik waren Rentner/innen, (Langzeit-)Arbeitslose, Sozialhilfebezieher/ innen, Studierende, Schüler/innen und die Familien von Geringverdiener(inne)n.

Dass die von 2009 bis 2013 amtierende Regierung aus CDU, CSU und FDP viele der hier kritisierten Maßnahmen an sozialer Härte noch überboten und manche Gesetze weiter verschärft hat, ${ }^{17}$ spricht nicht für eine Neuauflage der Großen Koalition. Nötig wäre nämlich eine umfassende Kurskorrektur auf zentralen Politikfeldern, insbesondere der Wirtschafts-, Steuer- und Finanzpolitik, der Sozial-, Arbeitsmarkt- und Beschäftigungspolitik, der Familien-, Bildungsund Gesundheitspolitik, aber auch der Wohnungsbau- und Stadtentwicklungspolitik, wie sie Sozialdemokrat(inn)en, Bündnisgrüne und LINKE im vergangenen Bundestagswahlkampf mit unterschiedlicher Akzent- bzw. Schwerpunktsetzung gefordert hatten.

15 Vgl. Christoph Butterwegge, Armut in einem reichen Land. Wie das Problem verharmlost und verdrängt wird, 3. Aufl. Frankfurt am Main/New York 2012, S. $234 \mathrm{ff}$.

16 Siehe Manfred G. Schmidt, Die Sozialpolitik der zweiten Großen Koalition (2005 bis 2009), in: Christoph Egle/Reimut Zohlnhöfer (Hrsg.), Die zweite Große Koalition, a.a.O., S. 317

17 Vgl. hierzu: Christoph Butterwegge, Krise und Zukunft des Sozialstaates, a.a.O, S. $289 \mathrm{ff}$. 